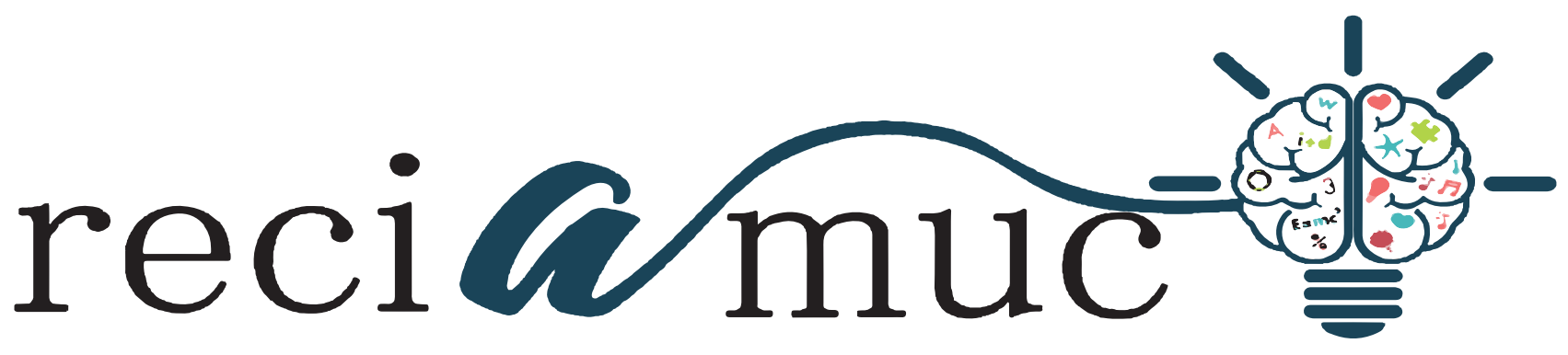

DOl: $10.26820 /$ reciamuc/5.(1).ene.2021.90-100

URL: https://reciamuc.com/index.php/RECIAMUC/article/view/596

EDITORIAL: Saberes del Conocimiento

REVISTA: RECIAMUC

ISSN: 2588-0748

TIPO DE INVESTIGACIÓN: Artículo de Revisión

CóDIGO UNESCO: 32 Ciencias Médicas

PAGINAS: 90-100

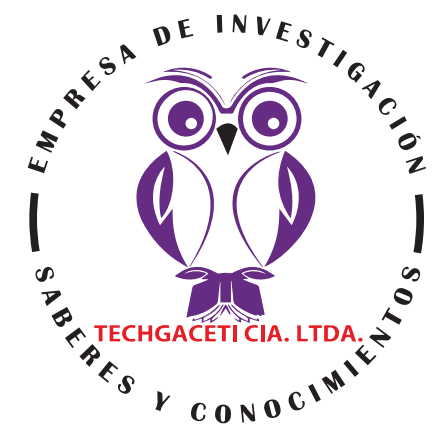

\title{
Hígado graso no alcohólico en niños obesos
}

Nonalcoholic fatty liver in obese children

Fígado gordo não alcoólico em crianças obesas

Tania Teresa Ortiz Jumbo'; Tasha Boriza Delgado Burgos²; Cisne Kaina Macías Hidrovo3; Erick Hugo Zambrano Franco ${ }^{4}$

RECIBIDO: 10/12/2020 ACEPTADO: 29/12/2020 PUBLICADO: 31/01/2021

1. Médico; Investigadora Independiente; Guayaquil, Ecuador; tania.ortizj@hotmail.com; (DD https://orcid.org/00000001-6211-9021

2. Médico; Investigadora Independiente; Guayaquil, Ecuador; tashadelgado15@gmail.com; (D) https://orcid. org/0000-0003-4225-1150

3. Médico; Investigadora Independiente; Guayaquil, Ecuador; kainamaciash@hotmail.com; (D) https://orcid. org/0000-0002-8828-9499

4. Médico; Investigador Independiente; Guayaquil, Ecuador; dr_ezambrano@hotmail.com; (D) https://orcid. org/0000-0001-6267-3022

CORRESPONDENCIA

Tania Teresa Ortiz Jumbo

tania.ortizj@hotmail.com

Guayaquil, Ecuador

๑ RECIAMUC; Editorial Saberes del Conocimiento, 2021 


\section{RESUMEN}

Aunque la enfermedad del hígado graso no alcohólico se identificó por primera hace unas cuantos decadas atrás, presenta una fisiopatología multifactorial y poco clara. El hígado graso no alcohólico (EHGNA), es una enfermedad caracterizada por la acumulación de grasa en el hígado que no es causada por el consumo de alcohol, donde las persona que lo padecen no tienen ni siquiera antecedentes de consumo de alcohol por lo cual este tipo de patología está asociada al sobrepeso. En esta revisión, se pretende actualizar los mecanismos fisiopatológicos de una enfermedad que presenta una alta incidencia de morbilidad y mortalidad y que está afectando a los niños obesos en todo el mundo. Se describe el conocimiento reciente sobre los diferentes mecanismos relacionados con la fisiopatología de la enfermedad del hígado graso no alcohólico centrado en los aspectos histológicos, anatómicos y bioquímicos implicados en el desencadenamiento de la esteatohepatitis (EHNA) y la cirrosis. La investigación clínica y los recursos tecnológicos avanzados han demostrado varios mecanismos fisiopatológicos determinantes de la enfermedad del hígado graso no alcohólico tratando de ayudar en su tratamiento y cambiar su curso natural.

Palabras clave: Hígado graso no alcohólico. Hígado graso. Obesidad.

\section{ABSTRACT}

Although nonalcoholic fatty liver disease was first identified a few decades ago, it has an unclear and multifactorial pathophysiology. Nonalcoholic fatty liver (NAFLD) is a disease characterized by the accumulation of fat in the liver that is not caused by alcohol consumption, where people who suffer from it do not even have a history of alcohol consumption, which is why this type of pathology is associated with being overweight. In this review, the aim is to update the pathophysiological mechanisms of a disease that has a high incidence of morbidity and mortality and that is affecting obese children worldwide. Recent knowledge on the different mechanisms related to the pathophysiology of nonalcoholic fatty liver disease is described, focusing on the histological, anatomical and biochemical aspects involved in the triggering of steatohepatitis (NASH) and cirrhosis. Clinical research and advanced technological resources have demonstrated several determining pathophysiological mechanisms of non-alcoholic fatty liver disease, trying to help in its treatment and change its natural course.

KeyWords: Non-alcoholic fatty liver. Fatty liver. Obesity.

\section{RESUMO}

Embora a doença não alcoólica do fígado gordo tenha sido identificada pela primeira vez há algumas décadas, tem uma patofisiologia pouco clara e multifactorial. O fígado gordo não alcoólico (NAFLD) é uma doença caracterizada pela acumulação de gordura no fígado que não é provocada pelo consumo de álcool, onde as pessoas que sofrem deste não têm sequer um historial de consumo de álcool, razão pela qual este tipo de patologia está associado ao excesso de peso. Nesta revisão, o objectivo é actualizar os mecanismos fisiopatológicos de uma doença que tem uma elevada incidência de morbilidade e mortalidade e que está a afectar crianças obesas em todo o mundo. São descritos conhecimentos recentes sobre os diferentes mecanismos relacionados com a fisiopatologia da doença não alcoólica do fígado gordo, focando os aspectos histológicos, anatómicos e bioquímicos envolvidos no desencadeamento da estetohepatite (NASH) e cirrose. A investigação clínica e os recursos tecnológicos avançados demonstraram vários mecanismos fisiopatológicos determinantes da doença hepática gorda não-alcoólica, tentando ajudar no seu tratamento e alterar o seu curso natural.

Palavras-chave: Fígado gordo não-alcoólico. Fígado gorduroso. Obesidade. 


\section{Introducción}

Los continuos avances médicos científicos de las últimas décadas proporcionan nuevos conocimientos sobre la fisiopatología de algunos problemas de salud intrigantes, como la enfermedad del hígado graso no alcohólico (EHGNA). Descrita por primera vez por Ludwig, Viggiano, McGill, \& Oh, (1980), la EHGNA "es una enfermedad caracterizada por la acumulación de grasa en el citoplasma de los hepatocitos, no relacionada con el alcohol, causando el llamado hígado graso".

La presencia de al menos el 5\% de los hepatocitos afectados en los fragmentos de las biopsias proporciona el diagnóstico histopatológico de hígado graso. "Esta afección se caracteriza por un espectro que va desde la esteatosis hepática hasta la esteatohepatitis (caracterizada por inflamación y fibrosis) y, finalmente, cirrosis" (Padilha, Rocha, Alves, \& Peres, 2010). Es, por tanto, una afección grave que puede provocar insuficiencia hepática en un corto período de tiempo.

La EHGNA es ahora la forma más común de enfermedad hepática crónica y su prevalencia está creciendo rápidamente en todo el mundo. La prevalencia real de EHGNA en la población pediátrica aún es desconocida y variable, con un rango de prevalencia del 3,0 al $60,3 \%$ en niños y adolescentes obesos.

Sus mecanismos fisiopatológicos aún no se conocen del todo, aunque se reconoce que está aumentando especialmente entre niños y adolescentes con sobrepeso, presentando al menos el 50\% de ellos algún grado de EHGNA. La prevalencia de EHGNA es variable según la región, la población encuestada y el modo de diagnóstico de EHGNA (Padilha, Rocha, Alves, \& Peres, 2010).

La evidencia disponible sobre la morbilidad y la mortalidad asociadas con la EHGNA demuestra que los niños afectados por la
EHGNA tienen un mayor riesgo de muerte o de someterse a un trasplante de hígado en comparación con los niños de la misma edad y sexo pero sin la enfermedad. "La historia natural de la EHGNA en los niños revela una evolución progresiva de la enfermedad, que conduce al desarrollo de cirrosis hepática en un porcentaje expresivo de esta población" (Schwimmer, Deutsch, \& Kahen, 2007)

En tal sentido, la EHGNA está estrechamente relacionada con la obesidad, los factores ambientales / nutricionales y la predisposición genética. La obesidad, un trastorno nutricional crónico y prevalente en el mundo, a menudo se asocia con hígado graso, hipertensión, resistencia a la insulina y dislipidemia. Afecta a varios grupos de edad, incluidos niños y adolescentes.

En un modelo murino, "la esteatosis inducida por obesidad condujo a un aumento del estrés oxidativo, así como a una inflamación crónica del hígado" (Schwimmer, Deutsch, \& Kahen, 2007). La resistencia a la insulina, una condición frecuente en pacientes obesos, tiene un papel clave en la fisiopatología de la EHGNA. De manera similar, la alta ingesta de alimentos común a la obesidad puede causar cambios en la flora intestinal, y la lesión del metabolismo de los lípidos causa hígado graso. Los hallazgos de laboratorio en individuos obesos mostraron niveles elevados de marcadores inflamatorios, proteínas de inflamación de fase aguda, hormonas, radicales libres y otros factores de activación endotelial. Este hecho demuestra la existencia de una afección inflamatoria subyacente que determina la aparición de EHGNA.

A través del desarrollo de la siguiente revisión bibliográfica, se describen datos actualizados y completos para explicar la fisiopatología de la EHGNA, especialmente en niños y adolescentes obesos. En esta población la evolución de la EHGNA es más insidiosa que en los adultos, probablemente debido a que este grupo de edad 
no presenta los mecanismos adecuados de adaptación a los cambios metabólicos provocados por la obesidad. Por otro lado, esta población es supuestamente más susceptible a intervenciones terapéuticas, lo que la convierte en candidata a nuevas estrategias de tratamiento para prevenir la evolución a enfermedad hepática terminal.

\section{Metodología}

Para el desarrollo de este proceso investigativo, se plantea como metodología la encaminada hacia una orientación científica particular que se encuentra determinada por la necesidad de indagar en forma precisa y coherente una situación, en tal sentido Davila, (2015) define la metodología "como aquellos pasos anteriores que son seleccionados por el investigador para lograr resultados favorables que le ayuden a plantear nuevas ideas" (p.66)

Lo citado por el autor, lleva a entender que el desarrollo de la acción investigativa busca simplemente coordinar acciones enmarcadas en una revisión bibliográfica con el fin de complementar ideas previas relacionadas Hígado graso no alcohólico en niños obesos a través de una revisión de literatura, para así finalmente elaborar un cuerpo de consideraciones generales que ayuden a ampliar el interés propuesto.

\section{Tipo de Investigación}

Dentro de toda práctica investigativa, se precisan acciones de carácter metodológico mediante las cuales se logra conocer y proyectar los eventos posibles que la determinan. En este sentido, la presente investigación corresponde al tipo documental, definido por Castro (2016), "se ocupa del estudio de problemas planteados a nivel teórico, la información requerida para abordarlos se encuentra básicamente en materiales impresos, audiovisuales y / o electrónicos". (p.41).

En consideración a esta definición, la orientación metodológica incluye la oportunidad de cumplir con una serie de actividades inherentes a la revisión y lectura de diversos documentos, donde se encuentran ideas explicitas relacionadas con los tópicos encargados de identificar una característica inmersa en el estudio. Por lo tanto, se realizaron continuas interpretaciones con el claro propósito de revisar aquellas apreciaciones propuestas por diferentes investigadores en relación al tema de interés, para luego dar la respectiva argumentación a los planteamientos, en función a las necesidades encontradas en la investigación, apoyados en las herramientas tecnológicas para la búsqueda de trabajos con valor científico disponibles en la web que tenían conexión con el objetivo principal de la investigación.

\section{Fuentes Documentales}

El análisis correspondiente a las características que predomina en el tema seleccionado, llevan a incluir diferentes fuentes documentales encargadas de darle el respectivo valor científico y en ese sentido cumplir con la valoración de los hechos a fin de generar nuevos criterios que sirven de referencia a otros procesos investigativos. Para Castro,(2016) las fuentes documentales incorporadas en la investigación documental o bibliográfica, "representa la suma de materiales sistemáticos que son revisados en forma rigurosa y profunda para llegar a un análisis del fenómeno" (p.41). Por lo tanto, se procedió a cumplir con la lectura previa determinada para encontrar aquellos aspectos estrechamente vinculados con el tema, con el fin de explicar mediante un desarrollo las respectivas apreciaciones generales de importancia.

\section{Técnicas para la Recolección de la Infor- mación}

La conducción de la investigación para ser realizada en función a las particularidades que determinan a los estudios documentales, tiene como fin el desarrollo de un conjunto de acciones encargadas de llevar a la selección de técnicas estrechamente vinculadas con las características del estudio.

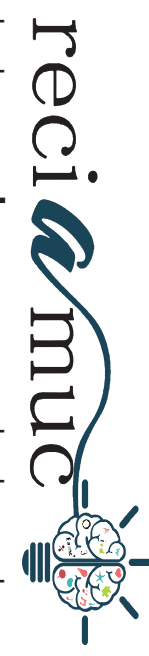


Bolívar, (2015), refiere, que es "una técnica particular para aportar ayuda a los procedimientos de selección de las ideas primarias y secundarias". (p.71).

Tal como lo expresa, Bolívar, (2015) "Las técnicas documentales proporcionan las herramientas esenciales y determinantes para responder a los objetivos formulados y llegar a resultados efectivos" (p. 58). Es decir, para responder con eficiencia a las necesidades investigativas, se introdujeron como técnica de recolección el método inductivo, que hizo posible llevar a cabo una valoración de los hechos de forma particular para llegar a la explicación desde una visión general. El autor Bolívar, (2015) tambien expresa que las técnicas de procesamiento de datos en los estudios documentales "son las encargadas de ofrecer al investigador la visión o pasos que deben cumplir durante su ejercicio, cada una de ellas debe estar en correspondencia con el nivel a emplear" (p. 123). Esto indica, que para llevar a cabo el procesamiento de los datos obtenidos una vez aplicadas las técnicas seleccionadas, tales como: fichas de resumen, textual, registros descriptivos entre otros, los mismos se deben ajustar al nivel que ha sido seleccionado.

\section{Resultados}

Con la tendencia creciente de la obesidad, la enfermedad del hígado graso no alcohólico (EHGNA) se ha convertido ahora en la causa más común de enfermedad hepática crónica en niños y adolescentes, "con una prevalencia del 3\% al 10\% en la población pediátrica general que aumenta hasta un 70\% en niños obesos" (Roberts, 2007).

La EHGNA es una entidad clínico-patológica que abarca un amplio espectro de lesiones hepáticas que van desde la acumulación de grasa en el hígado (esteatosis simple) hasta la forma potencialmente progresiva de esteatohepatitis no alcohólica (EHNA) caracterizada por hinchazón e inflamación de los hepatocitos, y a menudo se asocia con fibrosis. EHGNA puede causar cirrosis descompensada que requiere trasplante de hígado y carcinoma hepatocelular incluso en niños. (Roberts, 2007)

Aunque la EHGNA aumenta el riesgo de mortalidad y morbilidad relacionadas con el hígado, las causas más comunes de muerte entre los pacientes con EHGNA son las enfermedades cardiovasculares (ECV) y las neoplasias malignas extrahepáticas. Esto ha llevado a una mayor conciencia de las complicaciones extrahepáticas asociadas con EHGNA. La EHGNA "se considera una manifestación hepática del síndrome metabólico (MetS); sin embargo, los datos emergentes indican que EHGNA puede ser un factor de riesgo para el desarrollo de MetS, diabetes mellitus (DM) tipo 2 y ECV" (Miele \& Targher, 2015).

De manera similar, "se ha demostrado que la EHGNA se asocia con otras complicaciones extrahepáticas, como enfermedad renal crónica, hipotiroidismo, síndrome de ovario poliquístico, apnea obstructiva del sueño (AOS), osteoporosis y cáncer colorrectal en adultos" (Miele \& Targher, 2015). En los niños, la evidencia reciente sugiere que la EHGNA pediátrica se asocia con complicaciones extrahepáticas individuales, como ECV, DM tipo 2, retinopatía, deficiencia de vitamina $\mathrm{D}$ y baja densidad mineral ósea.

\section{Manifestaciones clínicas}

Los niños con EHGNA generalmente se diagnostican debido a una elevación accidental de las enzimas hepáticas o evidencia de esteatosis en la ecografía realizada como parte de una prueba de detección de rutina en niños obesos o para la evaluación de otras enfermedades. Los niños permanecen asintomáticos y se presentan clínicamente una vez que la enfermedad hepática ha progresado o con manifestaciones extrahepáticas concurrentes de MetS.

Se informa que la edad media de diagnóstico de EHGNA en niños es de 11 a 13 años. Las manifestaciones clínicas de EHGNA incluyen dolor abdominal inespecífico en 
el cuadrante superior derecho por estiramiento de la cápsula hepática (aproximadamente $42 \%-59 \%$ de los pacientes), fatiga e irritabilidad. El examen físico puede revelar acantosis nigricans por resistencia a la insulina (RI); hepatomegalia en hasta el 50\% de los pacientes, que puede ser difícil de evaluar debido a la obesidad abdominal; $y$, raramente, esplenomegalia (Vajro, Lenta, \& Socha, 2012).

\section{Métodos de diagnóstico Enzimas hepáti- cas}

A pesar de la alta prevalencia de EHGNA en niños, los enfoques de detección y diagnóstico en la EHGNA pediátrica no están bien definidos. De acuerdo (Schwimmer, Dunn, \& Norman, 2010)

La Academia Estadounidense de Pediatría recomienda la detección semestral de niños de 10 años o más que tienen sobrepeso con otros factores de riesgo de EHGNA u obesidad, incluso sin factores de riesgo con niveles de alanina aminotransferasa (ALT) y aspartato aminotransferasa (AST) y una posterior derivación al hepatólogo pediátrico si los niveles de ALT o AST son 2 veces el límite superior de los niveles normales. Sin embargo, la Sociedad Europea de Hepatología y Nutrición de Gastroenterología Pediátrica recomienda el cribado en niños obesos de 3 años o más con enzimas hepáticas y ecografía (p. 64).

El análisis de datos de la Encuesta Nacional de Examen de Salud y Nutrición entre 1999 y 2006 en el estudio SAFETY mostró que

Los percentiles 95 para ALT en niños con peso saludable, metabólicamente normales y sin enfermedad hepática fueron $26 \mathrm{U} / \mathrm{L}$ en niños y $22 \mathrm{U} / \mathrm{L}$ en niñas en comparación con la mediana del límite superior de la normalidad de $53 \mathrm{U} / \mathrm{L}$ (rango 30-90 U / L) utilizado en diferentes hospitales de niños en los Estados Unido (Schwimmer, Deutsch, \& Kahen, 2007).

Por lo tanto, se debe usar un límite más bajo de ALT para detectar EHGNA para mejorar su sensibilidad.

\section{Imágenes}

La ecografía es una herramienta de detección ampliamente utilizada para la esteatosis hepática con una sensibilidad de aproximadamente el $80 \%$ y una especificidad de aproximadamente el $50 \%$ al $60 \%$. Sin embargo, "la ecografía ha disminuido la sensibilidad en pacientes con esteatosis leve, además, el ultrasonido no puede distinguir con precisión entre esteatosis simple y EHNA o fibrosis" (Saadeh, Younossi, \& Remer, 2002).

Las técnicas de imagen más nuevas, como el parámetro de atenuación controlado y la resonancia magnética con fracción de grasa de densidad de protones, han demostrado ser más precisas para evaluar la esteatosis hepática. "Recientemente se han desarrollado técnicas de imagen que miden la rigidez del hígado mediante elastografía para evaluar la fibrosis hepática" (Saadeh, Younossi, \& Remer, 2002). Aunque estas técnicas de imagen recientes son más precisas que la ecografía convencional, su uso es actualmente limitado debido al costo y la falta de valores de corte validados en niños.

\section{Biopsia hepática}

La biopsia hepática sigue siendo el estándar de oro en la evaluación de la esteatosis, la EHNA y la fibrosis hepática relacionada con la EHGNA. "La esteatosis simple se define como la esteatosis macro vesicular en el $5 \%$ de los hepatocitos después de excluir otras causas de esteatosis hepática, como la hepatitis viral, la enfermedad de Wilson o la hepatitis autoinmune" (Yeh, Brunt, \& Day, 2014). La EHNA se caracteriza por lesión de los hepatocitos (globo) e infiltración neutrofílica del hígado (inflamación lobulillar y portal).

En un análisis retrospectivo de niños con EHGNA comprobada por biopsia, se identificaron 2 patrones distintivos de histología. 
La esteatosis, la degeneración en globo, la inflamación lobular y la fibrosis perisinusoidal se clasificaron como EHNA tipo 1 (tipo adulto), mientras que la esteatosis, la inflamación portal y la fibrosis portal se clasificaron como EHNA tipo 2 (tipo pediátrico). Se descubrió que la EHNA tipo 2 es el patrón histológico más común que se observa en los niños más pequeños con EHGNA (Schwimmer, Dunn, \& Norman, 2010)

\section{Progresión de la enfermedad hepática}

Existen datos limitados sobre la progresión de la EHGNA pediátrica desde la esteatosis simple hasta la EHNA, la fibrosis y la cirrosis. Se ha demostrado que "la presencia de fibrosis hepática avanzada es un predictor de la mortalidad general y relacionada con el hígado, independientemente de otras características histológicas. La prevalencia de fibrosis hepática avanzada en niños con EHGNA es variable" (Schwimmer, Deutsch, \& Kahen, 2007).

En un estudio realizado por Alkhouri, Sedki, \& Alisi, (2013) "se encontró que solo el $15 \%$ de 67 niños con EHGNA comprobada por biopsia tenían fibrosis significativa (estadio 2-3)". En una revisión retrospectiva de la histología hepática de 742 niños a los que se les realizó una autopsia por muerte súbita esperada, "se observó EHNA en aproximadamente el $23 \%$ de los niños con hígado graso, de los cuales solo el 9\% tenían fibrosis en puente o cirrosis" (Schwimmer, Deutsch, \& Kahen, 2007). Por lo tanto, aproximadamente del $10 \%$ al $25 \%$ de los niños diagnosticados con EHGNA pueden progresar a fibrosis avanzada.

Múltiples estudios en adultos han demostrado que EHGNA es un factor de riesgo para el desarrollo de carcinoma hepatocelular $(\mathrm{CHC})$ incluso en ausencia de cirrosis. "Uno de los estudios de seguimiento en adultos con cirrosis mostró que la incidencia acumulada anual de $\mathrm{CHC}$ en pacientes con cirrosis EHNA fue del 2,6\%" (Ascha, Hanouneh, \& Lopez, 2010). Se ha informado de $\mathrm{CHC}$ en un paciente pediátrico en el con- texto de obesidad y esteatosis sin evidencia de fibrosis o cirrosis.

La EHGNA pediátrica podría conducir a un mayor riesgo de $\mathrm{CHC}$ en la edad adulta, pero esta asociación no se ha estudiado. El desarrollo de $\mathrm{CHC}$ en ausencia de cirrosis o fibrosis podría indicar el papel potencial de otros factores, como el síndrome metabólico, la obesidad, la RI o el estrés oxidativo en la patogenia del HCC en pacientes con EHGNA (Ascha, Hanouneh, \& Lopez, 2010).

\section{Complicaciones extrahepáticas}

Los mecanismos involucrados en el desarrollo de complicaciones extrahepáticas en niños con EHGNA no se comprenden completamente. Se hipotetiza que es causado por una interacción de muchos factores como mediadores proinflamatorios, estrés oxidativo, RI y lipotoxicidad. 
Fig. 1. Complicaciones extrahepáticas en niños con EHGNA.

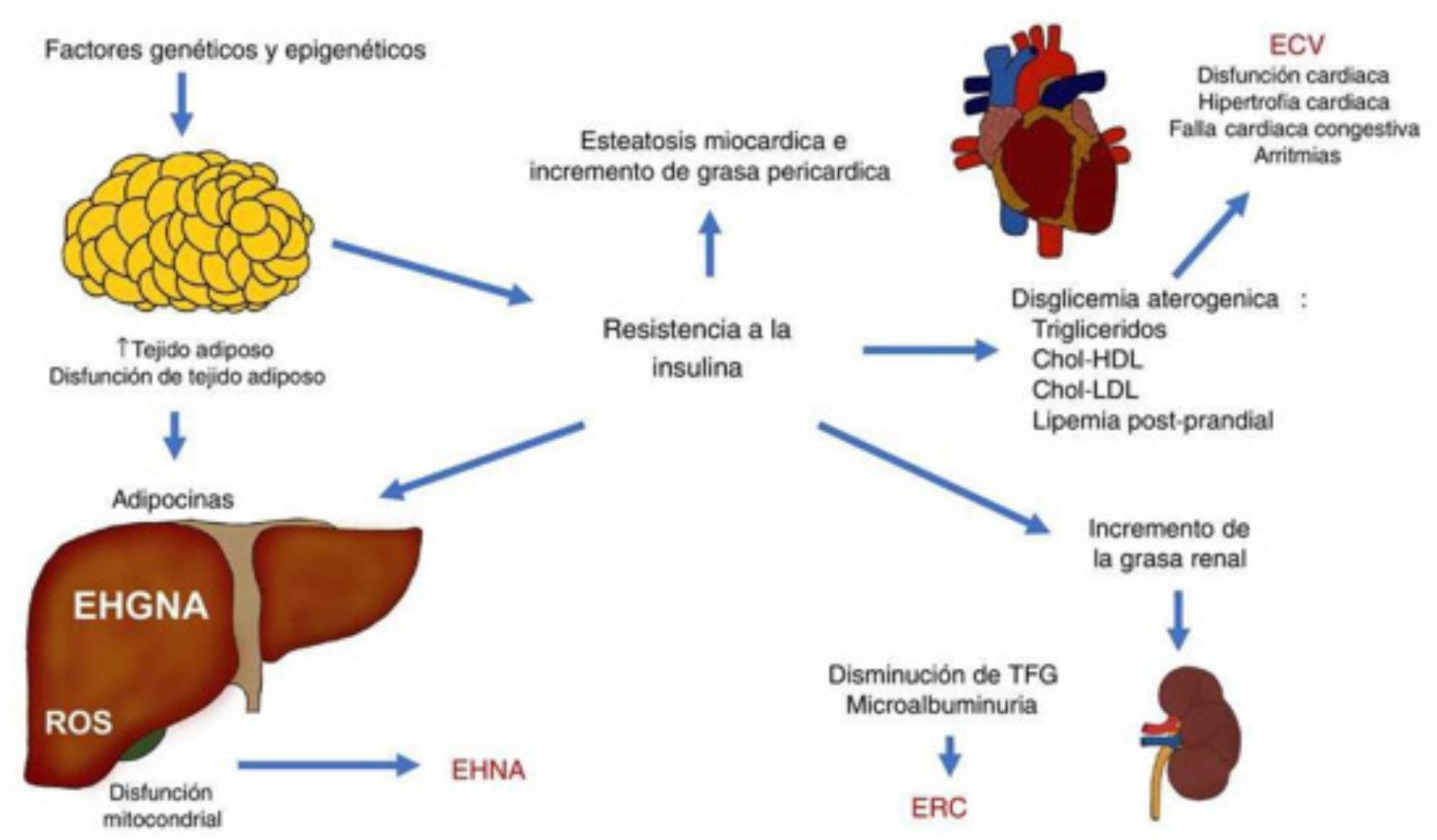

Fuente. (Calleja, Crespo, Raña, \& Rizo, 2019).

\section{Enfermedad cardiovascular}

En los últimos años, ha existido un gran interés en comprender la asociación entre EHGNA y la enfermedad cardiovascular (ECV) y el papel potencial de EHGNA en la fisiopatología de los cambios cardiovasculares. La evidencia de múltiples estudios en adultos "sugiere que la EHGNA es un factor de riesgo independiente de ECV y se ha encontrado que se asocia con disfunción endotelial, aumento del grosor de la íntima carotídea y mayor prevalencia de placas de arterias coronarias" (Schwimmer, Deutsch, \& Kahen, 2007). Sin embargo, los estudios que evalúan la ECV en la EHGNA pediátrica son limitados.

En un estudio de casos y controles, los niños obesos con EHGNA comprobada por biopsia tenían colesterol total (CT), colesterol unido a lipoproteínas de baja densidad (LDL-C), triglicéridos (TG), glucosa en ayunas y presión arterial significativamente más altos que los niños con obesidad sola, lo que indica un mayor perfil de riesgo car- diovascular en niños con EHGNA (Schwimmer, Deutsch, \& Kahen, 2007).

La aterosclerosis puede comenzar ya en la infancia, con el depósito de vetas grasas en las arterias coronarias y carótidas. "La evaluación de la aterosclerosis subclínica y el riesgo de ECV en niños se puede lograr con el perfil lipídico y el examen de las estructuras vasculares, como el grosor de la íntima media carotídea (CIMT) o la disfunción endotelial" (Nobili, Alkhouri, \& Bartuli, 2010)

\section{Diabetes mellitus tipo 2 y metabolismo anormal de la glucosa}

La RI juega un papel importante en la patogenia de la EHGNA y, por tanto, el metabolismo anormal de la glucosa es muy frecuente entre los pacientes con EHGNA.

Múltiples estudios en adultos informaron probabilidades significativamente más altas de desarrollar DM tipo 2 en pacientes con EHGNA.10 También se ha demostrado que la DM tipo 2 es un factor de riesgo inde- 
pendiente para la progresión de EHGNA a EHNA y fibrosis avanzada.49 Se ha demostrado que la esteatosis hepática en niños aumenta el riesgo de IR y desregulación de la glucosa (D’Adamo, Cali, \& Weiss, 2010)

Un hallazgo clave es que los niños con prediabetes y diabetes tenían probabilidades significativamente más altas de desarrollar EHNA. Así, "estudios pediátricos de reciente aparición indican que la DM tipo 2 es un factor de riesgo para la progresión de la enfermedad hepática en la EHGNA con un posible aumento de la mortalidad y morbilidad relacionadas con el hígado" (D’Adamo, Cali, \& Weiss, 2010). Se necesitan estudios longitudinales para comprender la relación causa-efecto entre EHGNA y DM tipo 2.

\section{Deficiencia de vitamina D}

La deficiencia de vitamina $D$ se ha asociado con la obesidad en adultos y niños. Se encuentra que los pacientes adultos con EHGNA tienen una alta prevalencia de deficiencia de vitamina $D$ con niveles bajos de vitamina $\mathrm{D}$, lo que se correlaciona con la gravedad histológica de EHGNA.

De manera similar, "los estudios pediátricos también han informado una alta prevalencia en niños con EHGNA, pero la correlación con la gravedad histológica es contradictoria" (Targher, Bertolini, \& Scala, 2007). Además, "los niveles bajos de vitamina $D$ también se correlacionaron con la gravedad de la fibrosis hepática" (Nobili, Giorgio, \& Liccardo, 2014).

\section{Osteopenia y osteoporosis}

La osteoporosis es más frecuente en pacientes con enfermedad hepática crónica. Existe evidencia acumulada para respaldar el impacto de EHGNA en la salud ósea tanto en adultos como en niños. La relación entre la densidad mineral ósea (DMO) y la EHGNA pediátrica se evaluó por primera vez en un estudio turco en el que participaron niños obesos con o sin esteatosis hepática (diagnosticada con ecografía hepá- tica). "Los niños con esteatosis hepática en la ecografía tenían puntuaciones Z de DMO en la columna más bajas en comparación con los niños sin esteatosis hepática" (Pirgon, Bilgin, \& Tolu, 2011). A pesar de estos hallazgos, el papel de EHGNA en la osteoporosis y el riesgo de fracturas en niños con EHGNA no se comprende claramente debido a la falta de estudios longitudinales.

\section{Apnea obstructiva del sueño}

La AOS ha sido reconocida como un factor de riesgo para EHGNA, EHNA y fibrosis independientemente de la edad, el sexo y el IMC en adultos. "En 2 estudios pediátricos, la evaluación polisomnografíca de niños con EHGNA comprobada por biopsia mostró una prevalencia de aproximadamente el $60 \%$. Además, la AOS se asoció significativamente con la EHNA y la gravedad de la fibrosis hepática" (Nobili, Giorgio, \& Liccardo, 2014). Se postula que la progresión de la EHGNA en el contexto de la AOS podría deberse a hipoxemia, que puede crear un estrés oxidativo, o hipoxemia y normoxia alternas, que podría producir una lesión de tipo isquémico-reperfusión en el hígado. La AOS puede afectar significativamente el rendimiento escolar y los niveles de actividad de los niños, por lo que es importante realizar pruebas de detección de la AOS en los niños con EHGNA.

\section{Calidad de vida}

Con el aumento de las comorbilidades asociadas con EHGNA, los pacientes con EHGNA pueden tener una mala calidad de vida (QOL). La comparación de los datos de calidad de vida entre adultos con EHGNA y la población con y sin enfermedad crónica muestra peores puntuaciones de salud física y mental en pacientes con EHGNA. Además, una puntuación de salud física más baja se asoció con la gravedad de la enfermedad hepática entre los pacientes con EHGNA. Pocos estudios pediátricos también han abordado los problemas psicosociales en niños con EHGNA. Una mala calidad de vida en la EHGNA pediátrica puede 
aumentar la carga de enfermedad tanto en los niños como en los padres. Por lo tanto, es importante detectar problemas psicosociales y abordarlos en consecuencia.

\section{Conclusiones}

Aunque la EHGNA es una de las principales causas de enfermedad hepática crónica en niños y adolescentes de países desarrollados, varios aspectos de la EHGNA pediátrica siguen sin estar claros. La mayoría de los estudios pediátricos sobre EHGNA son transversales con un tamaño de muestra limitado. Esto limita la comprensión de la historia natural de la enfermedad en EHGNA.

A pesar de la carga significativa de la enfermedad y su potencial de progresión a cirrosis incluso en niños y adultos jóvenes, todavía faltan métodos de detección bien establecidos. Los biomarcadores no invasivos y las técnicas de imagen en la evaluación de la EHGNA se están estudiando ampliamente, pero falta la validación de estas herramientas. Además, las opciones de tratamiento para la enfermedad relacionada con el hígado en la EHGNA pediátrica son limitadas. Actualmente, la modificación del estilo de vida, incluidos los hábitos alimentarios saludables, la pérdida de peso y la actividad física es lo que mayormente se recomienda para tratar esta enfermedad.

Durante el desarrollo del proceso investigativo se observó, que múltiples estudios en adultos y pediátricos han ampliado el espectro de EHGNA para incluir numerosas complicaciones extrahepáticas. Sin embargo es evidente que se necesitan más estudios longitudinales prospectivos a largo plazo para comprender la compleja interacción de los factores involucrados en el desarrollo de estas complicaciones extrahepáticas. Por lo tanto, es importante que los médicos reconozcan estas complicaciones asociadas con EHGNA pediátrica para lo cual es necesario establecer pautas adecuadas para la detección de estas complicaciones en niños con EHGNA, ya que esto podría tener un efecto sobre la morbilidad y mortalidad a largo plazo de la enfermedad.

\section{Bibliografía}

Alkhouri, N., Sedki, E., \& Alisi, A. (2013). Combined paediatric NAFLD fibrosis index and transient elastography to predict clinically significant fibrosis in children with fatty liver disease. Liver Int , 79-85.

Ascha, M., Hanouneh, I., \& Lopez, R. (2010). The incidence and risk factors of hepatocellular carcinoma in patients with nonalcoholic steatohepatitis. Hepatology , 1972-8.

Bolívar, J. (2015). Investigación Documental. México. Pax.

Calleja, J., Crespo, J., Raña, R., \& Rizo, M. (2019). Diagnóstico y tratamiento de la enfermedad hepáticapor depósito de grasa. Una aproximación multidisciplinaren hígado graso. Rev Gastroenterologia de Mexico , 84 (82), 472-81.

Castro, J. (2016). Técnicas Documentales. México. Limusa.

Corey, K., Vuppalanchi, R., \& Vos, M. (2015). Improvement in liver histology is associated with reduction in dyslipidemia in children with nonalcoholic fatty liver disease. J Pediatr Gastroenterol Nutr , 360-7.

D’Adamo, E., Cali, A., \& Weiss, R. (2010). Central role of fatty liver in the pathogenesis of insulin resistance in obese adolescents. Diabetes Care , 1817-22.

Davila, A. (2015). Diccionario de Términos Científicos. . Caracas: Editorial Oasis.

Ludwig, J., Viggiano, T., McGill, D., \& Oh, B. (1980). Nonalcoholic steatohepatitis: Mayo Clinic experiences with a hitherto unnamed disease. Mayo Clin Proc , 434-8.

Miele, L., \& Targher, G. (2015). Understanding the association between developing a fatty liver and subsequent cardio-metabolic complications. Expert Rev Gastroenterol Hepatol , 1243-5.

Nobili, V., Alkhouri, N., \& Bartuli, A. (2010). Severity of liver injury and atherogenic lipid profile in children with nonalcoholic fatty liver disease. Pediatr Res , 665-70.

Nobili, V., Giorgio, V., \& Liccardo, D. (2014). Vitamin $D$ levels and liver histological alterations in children with nonalcoholic fatty liver disease. Eur $J$ Endocrinol , 547-53.

Padilha, P., Rocha, H., Alves, N., \& Peres, W. (2010). Prevalence of nonalcoholic fatty liver disease in obese children and adolescents: A systematic re-

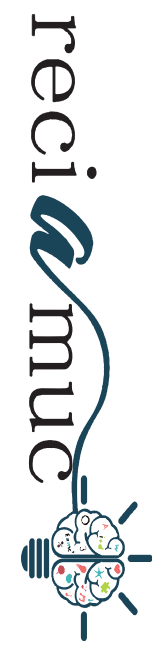


view. Rev Paul Pediatr , 387-93.

Pirgon, O., Bilgin, H., \& Tolu, I. (2011). Correlation of insulin sensitivity with bone mineral status in obese adolescents with nonalcoholic fatty liver disease. Clin Endocrinol , 189-95.

Roberts, E. (2007). Pediatric nonalcoholic fatty liver disease (NAFLD): a "growing" problem? J Hepatol , 133-42.

Saadeh, S., Younossi, Z., \& Remer, E. (2002). The utility of radiological imaging in nonalcoholic fatty liver disease. Gastroenterology , 745-50.

Schwimmer, J., Deutsch, R., \& Kahen, T. (2007). Prevalence of fatty liver in children and adolescents. Pediatrics, 118:138.
Schwimmer, J., Dunn, W., \& Norman, G. (2010). SAFETY study: alanine aminotransferase cutoff values are set too high for reliable detection of pediatric chronic liver disease. Gastroenterology , 357-64.

Targher, G., Bertolini, L., \& Scala, L. (2007). Associations between serum 25-hydroxyvitamin D3 concentrations and liver histology in patients with non-alcoholic fatty liver disease. Nutr Metab Cardiovasc Dis , 517-24.

Vajro, P., Lenta, S., \& Socha, P. (2012). Diagnosis of nonalcoholic fatty liver disease in children and adolescents. J Pediatr Gastroenterol Nutr , 700-13.

Yeh, M., Brunt, E., \& Day, C. (2014). Pathological features of fatty liver disease. Gastroenterology , 754-64.

\section{CITAR ESTE ARTICULO:}

Ortiz Jumbo, T. T., Delgado Burgos, T. B., Macías Hidrovo, C. K., \& Zambrano Franco, E. H. (2021). Hígado graso no alcohólico en niños obesos. RECIAMUC, 5(1), 90-100. https://doi.org/10.26820/reciamuc/5.(1).ene.2021.90-100

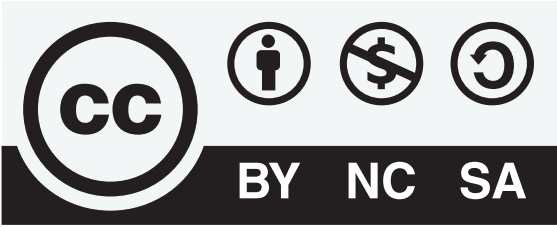

CREATIVE COMMONS RECONOCIMIENTO-NOCOMERCIAL-COMPARTIRIGUAL 4.0. 